\title{
A Novel Approach for the Solution of a Love's Integral Equations Using Bernstein Polynomials
}

\author{
Jumah Aswad Zarnan \\ Asst. Prof. Dr., Dept. Of Accounting by IT, Cihan University \Campus \Sulaimaniya, Kurdistan Iraq
}

\begin{abstract}
In this paper a novel technique implementing Bernstein polynomials is introduced for the numerical solution of a Love's integral equations. The Love's integral equation is a class of second kind Fredholm integral equations, and it can be used to describe the capacitance of the parallel plate capacitor (PPC) in the electrostatic field.This numerical technique developed by Huabsomboon et al. bases on using Taylor-series expansion [1], [2], [3] and [4]. We compare the numerical solution using Bernstein technique with the numerical solution obtained by using Chebyshev expansion. It is shown that the numerical results are excellent.

Keywords: Love's integral equation, Fredholm integral equation, Bernstein polynomials method, approximate solution.
\end{abstract}

\section{Introduction}

The topics of integral equations have been an increasing interest in the past years, because these kinds of equations appear in various fields of applied science and engineering. So, getting solutions with a high level of accuracy for the integral equations is a very important task. Considering that many real-world mathematical problems, especially in the area of applied mathematics are too complicated to be solved in exact terms, the using of numerical methods has been swiftly developed recently. Love's integral equation (Fredholm equation of the second kind) [5-14] has shown a big interest in the several applied physics fields such as polymer structures, aerodynamics, fracture mechanics hydrodynamics and elasticity engineering. For literature related to the numerical solutions of singular integral equations of the deterministic type, a surveys of different analytical methods for the solution of random integral equations has been proposed by Bharucha-Reid [5] and Christensen et al. [6]. Love's equations were early established by Love [10-12,15-18] for solving some magnetic and electrical fields problems. The actual study is concerned with calculation of the normalized field created conjointly by two similar plates of radius $\mathrm{R}$, separated by a distance $k \times R$, where $k$ is a positive real parameter, and at equal or opposite potential, with zero potential at infinity, is the solution of the Love's [16-17] second kind integral equation:

$u(x)=g(x)+\int_{-2}^{+2} K(x, t) u(t) d t$

Where $u$ is the normalized field to be determined, $g(x)$ is a given function and $K(x, t)$ is the rational kernel function with values in $[-1 ; 1]$ and defined by:

$K(x, t)= \pm \frac{1}{\pi} \frac{k}{k^{2}+(x-t)^{2}}$

Where the sign \pm codes for equal or opposite potential cases.

\section{Bernstein Polynomials}

The general form of the Bernstein polynomials [19] of nth degree over the interval [a,b] is defined by:

$B_{i, n}(x)=\left(\begin{array}{l}n \\ i\end{array}\right) \frac{(x-a)^{i}(b-x)^{n-i}}{(b-a)^{n}}, \quad a \leq x \leq b, i=0,1,2, \ldots, n$

Note that each of these $n+1$ polynomials having degree $n$ satisfies the following properties:

1. $B_{i, n}(x)=0$ if $i<0$ or $i>n$

2. $\sum_{i=0}^{n} B_{i, n}(x)=1$

3. $B_{i, n}(a)=B_{i, n}(b)=0, \quad 1 \leq i \leq n-1$

Using MATHEMATICA code, the first 11 Bernstein polynomials of degree ten over the interval $[a, b]$, are given below: 


$$
\begin{aligned}
& B_{0,10}(x)=(b-x)^{10} /(b-a)^{10} \\
& B_{1,10}(x)=10(b-x)^{9}(x-a) /(b-a)^{10} \\
& B_{2,10}(x)=45(b-x)^{8}(x-a)^{2} /(b-a)^{10} \\
& B_{3,10}(x)=120(b-x)^{7}(x-a)^{3} /(b-a)^{10} \\
& B_{4,10}(x)=210(b-x)^{6}(x-a)^{4} /(b-a)^{10} \\
& B_{5,10}(x)=252(b-x)^{5}(x-a)^{5} /(b-a)^{10} \\
& B_{6,10}(x)=210(b-x)^{4}(x-a)^{6} /(b-a)^{10} \\
& B_{7,10}(x)=120(b-x)^{3}(x-a)^{7} /(b-a)^{10} \\
& B_{8,10}(x)=45(b-x)^{2}(x-a)^{8} /(b-a)^{10} \\
& B_{9,10}(x)=10(b-x)(x-a)^{9} /(b-a)^{10} \\
& B_{10,10}(x)=(x-a)^{10} /(b-a)^{10}
\end{aligned}
$$

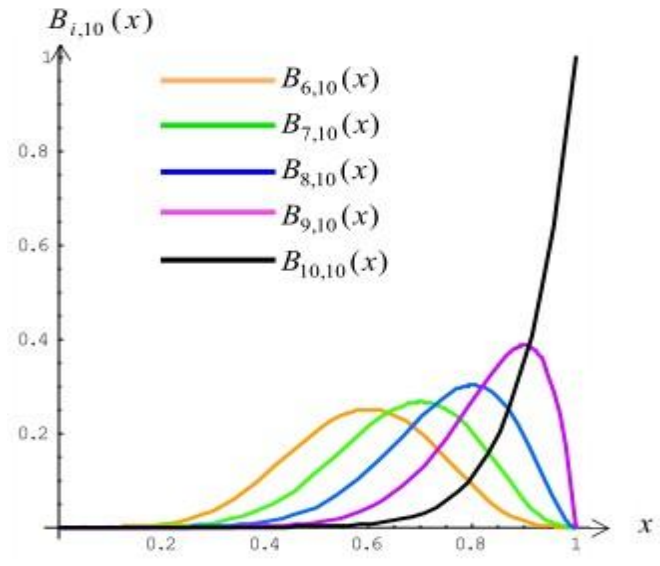

Fig. 1(b): Graph of last 5 Bernstein polynomials over $[0,1]$

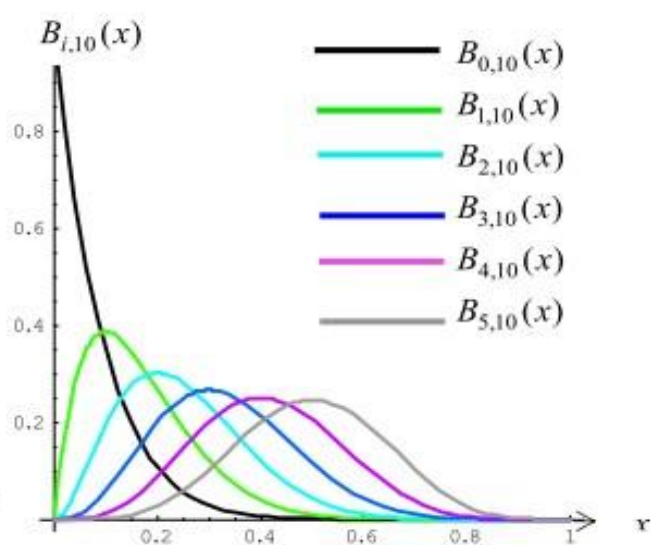

Fig. 1(a): Graph of first 6 Bernstein polynomials over $[0,1]$

Fig. 1(a), and the remaining five polynomials are shown in Fig. 1(b)Now the first six polynomials over $[0,1]$ are shown in

\section{Solution of A Love's Integral Equations}

In this section, first we consider the Love's integral equation (LIE) of the second kind given by [20, 21]:

$u(x) \pm \frac{1}{\pi} \int_{-1}^{1} K(x, t) u(t) d t=1 \quad-1 \leq x \leq 1$

here $u(x)$ is the unknown functions to be determined, $(x, t)=\frac{k}{k^{2}+(x-t)^{2}}$, the kernel is a continuous function. To determine an approximate solution of $(4) u(x)$ is approximated in the Bernstein polynomial basis on $[0,1]$ as:

$u(x)=\sum_{i=0}^{n} a_{i} B_{i, n}(x)$

Where $a_{i}, i=0,1, \ldots, n$ are unknown constants to be determined using Newton-Raphson method. Substituting (5) in (4), we obtain:

$\sum_{i=0}^{n} a_{i} B_{i, n}(x) \pm \frac{1}{\pi} \int_{-1}^{1} K(x, t) \sum_{i=0}^{n} a_{i} B_{i, n}(t) d t=1$ 
The linear system (6) can be solved by standard methods for the unknown constant $a_{I}$ 's.These $a_{i}, i=0,1, \ldots, n$ are then used in (5) to obtain the unknown function $u(x)$ approximately.

\section{Numerical Examples}

In this section, the method presented in this paper is used to find numerical solution of two illustrative examples. The solution of the equations obtained here. All calculations in the following tables are performed using Matlab. Example1. Consider the following Love's integral equation [15]

$u(x)-\frac{1}{\pi} \int_{-1}^{1} \frac{u(t)}{1+(x-t)^{2}} d t=1 \quad-1 \leq x \leq 1$

Where $u(x)$ is a real and continuous.The numerical result for $u(x)$ where $0 \leq x \leq 1$ is shown in Table 3 . Our approximate solutions agree well with Chebyshev series expansion method [22]. We observe that using $n=4$ obtain a good approximate solution as well as the absolute error.

Table 1: The approximate solution for $\mathrm{u}(\mathrm{x})$ in Example 1 with $\mathrm{n}=4$.

\begin{tabular}{|l|l|l|l|}
\hline $\mathrm{x}$ & Chebyshev Approx. & Our Approx. & Error \\
\hline 0.0 & 1.91903 & 1.91924 & $2.10 \mathrm{E}-04$ \\
\hline 0.1 & 1.91592 & 1.91557 & $3.50 \mathrm{E}-04$ \\
\hline 0.2 & 1.90659 & 1.90676 & $1.70 \mathrm{E}-04$ \\
\hline 0.3 & 1.89112 & 1.89113 & $1.00 \mathrm{E}-05$ \\
\hline 0.4 & 1.86964 & 1.86990 & $2.60 \mathrm{E}-04$ \\
\hline 0.5 & 1.84238 & 1.84733 & $4.95 \mathrm{E}-03$ \\
\hline 0.6 & 1.80974 & 1.80946 & $2.80 \mathrm{E}-04$ \\
\hline 0.7 & 1.77227 & 1.77161 & $6.60 \mathrm{E}-04$ \\
\hline 0.8 & 1.73075 & 1.73247 & $1.72 \mathrm{E}-03$ \\
\hline 0.9 & 1.68617 & 1.68674 & $5.70 \mathrm{E}-04$ \\
\hline 1.0 & 1.63969 & 1.54340 & $9.63 \mathrm{E}-02$ \\
\hline
\end{tabular}

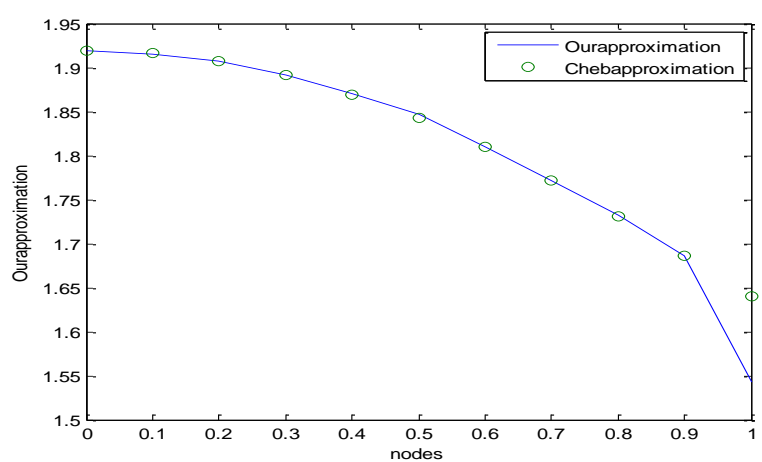

Fig. 2: The approximate solution for $u(x)$ in Example 1.

Example2. Consider the following Love's integral equation

$u(x)+\frac{1}{\pi} \int_{-1}^{1} \frac{u(t)}{1+(x-t)^{2}} d t=1 \quad-1 \leq x \leq 1$

Table 2: The approximate solution for $u(x)$ in Example 2 with $\mathrm{n}=4$.

\begin{tabular}{|l|l|l|l|}
\hline $\mathrm{X}$ & Chebyshev Approx. & Our Approx. & Error \\
\hline 0.0 & 0.65741 & 0.65708 & $3.30 \mathrm{E}-04$ \\
\hline 0.1 & 0.65844 & 0.65848 & $4.00 \mathrm{E}-05$ \\
\hline 0.2 & 0.66151 & 0.66151 & $0.00 \mathrm{E}+00$ \\
\hline 0.3 & 0.66666 & 0.66611 & $5.50 \mathrm{E}-04$ \\
\hline 0.4 & 0.67389 & 0.67382 & $7.00 \mathrm{E}-05$ \\
\hline 0.5 & 0.68318 & 0.68319 & $1.00 \mathrm{E}-05$ \\
\hline 0.6 & 0.69448 & 0.69429 & $1.90 \mathrm{E}-04$ \\
\hline 0.7 & 0.70766 & 0.70765 & $1.00 \mathrm{E}-05$ \\
\hline 0.8 & 0.72249 & 0.72224 & $2.50 \mathrm{E}-04$ \\
\hline 0.9 & 0.73865 & 0.73813 & $5.20 \mathrm{E}-04$ \\
\hline 1.0 & 0.75572 & 0.75506 & $6.60 \mathrm{E}-04$ \\
\hline
\end{tabular}




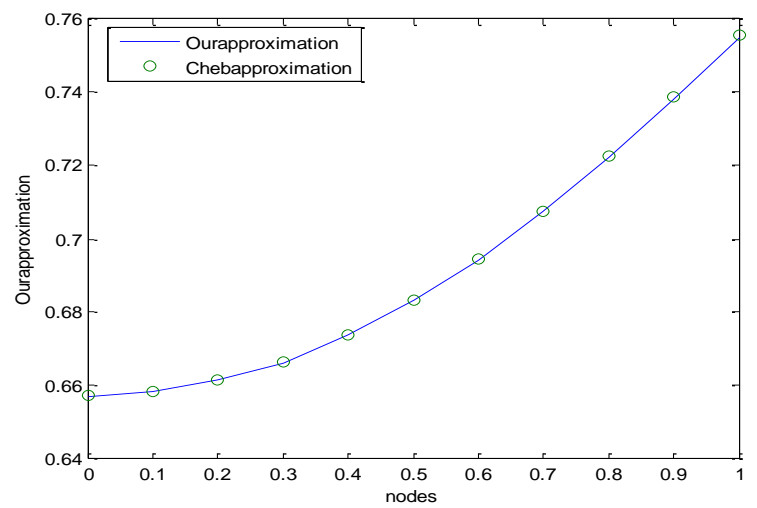

Fig. 3: The approximate solution for $u(x)$ in Example 2.

\section{Conclusion}

In this paper, we presented a useful numerical method that originated mainly from the Bernstein polynomials for solving Love's integral equation. As we explained above, this method converts the present problem to a system of linear algebraic equations which may be solvable easily. Having determined the unknown Bernstein coefficients of the solution function, the series solution is produced for numerical purposes immediately. It is important to be noted that, the more terms must be evaluated to the higher accuracy level. The obtained numerical results from analyzed examples illustrated that in applications involving computations with polynomials, the Bernstein form offers an efficient algorithm for many basic functions.

\section{References}

[1]. P. Huabsomboon, B. Novaprateep and H. Kaneko, On Taylor-series expansion methods for the second kind Integral equations, J. Comput. Appl. Math. 234, 2010, pp. 1466-1472.

[2]. P. Huabsomboon, B. Novaprateep and H. Kaneko, Taylor-series expansion methods for nonlinear Hammerstein equations. (to be appeared in Sci. Math. Jap.)

[3]. P. Huabsomboon, B. Novaprateep and H. Kaneko, Taylor-series expansion method for Volterra Integral Equations of the Second Kind, Sci. Math. Jap. 73, 2011,pp. 19-29.

[4]. P. Huabsomboon, B. Novaprateep and H. Kaneko, Discrete Taylor-expansion method for Integral Equations, Int. J. Numer. Appl. 1, 2009, pp. 139-153.

[5]. A. T. Bharucha-Reid, Approximation Solution of Random Equations. North Holland, New York (1979).

[6]. M. J. Christenscn, A. T. Bharucha-Reid,. J. Integ. Eq. 3, (1981) 217.

[7]. $\quad$ M. A. Golberg, J. Integ .Eq. 5 (1983) 330.

[8]. R. E. Scarton, Math. Comp. 23 (1969), 837

[9]. F. Erdogan, G. D. Gupta, T. S. Cook, Methods of Analysis and Solution to Crack Problems, NY (1973).

[10]. M. Sambandham, M. J. Christensen, A. T. Bharucha-Reid, Stoch. Analysis Applic. 3 (1985) 467

[11]. M. Sambandham, T. Srivatsan, A. T. Bharucha-Reid, Integral Methods in Science and Engineering, Eds. F. D. Payne et al (1986)

[12]. M. Sambandham, J. V. Thangaraj, K.B. Bota, , Comput. Math. Applic. 16 (1988) 915

[13]. B. Patel, A. Majethiya, P. C. Vinodkumar, Pramana-J. Phys. 72 (2009) 679

[14]. M. J. Christenscn and A. T. Bharucha-geid, J. Integ. Equation 3 (1981) 333.

[15]. E. R. Love, Quart. J. Mech. Appl. Math. 2 (1949) 428.

[16]. E. R. Love, Mathematika 37 (1990) 217.

[17]. Y. Ren, B. Zhang, H. Qiao, J. Comput. Appl. Math. 110 (1999)15.

[18]. D. F. Bartlett, R. Corle, J. Phys. A 18 (1985) 1337

[19]. B. N. Mandal and S. Bhattacharya, Appl. Math.Comput. 190, 1707 (2007).

[20]. E.R. Love, The electrostatic field of two equal circular co-axial conducting disks,Quart.J.Mech.Appl.Math.2 (1949), 428-451.

[21]. E.R.Love, The potential due to a circular parallel plate condenser, Mathematika37 (1990), $217-231$.

[22]. D. Elliott, A Chebyshev series method for the numerical solution of Fredholm integral equations, Comput. J. 6, 1963, pp. 102-111. 